\title{
Two dimensional inverse scattering from buried magnetic anomalies
}

\author{
Raffaele Persico $\left({ }^{1}\right)$ and Francesco Soldovieri $\left({ }^{2}\right)$ \\ (') Istituto per i Beni Archeologici e Monumentali (IBAM-CNR), Lecce, Italy \\ $\left.{ }^{(}{ }^{2}\right)$ Istituto per il Rilevamento Elettromagnetico dell'Ambiente (IREA-CNR), Napoli, Italy
}

\begin{abstract}
This paper deals with the problem of the electromagnetic linear inverse scattering from magnetic anomalies buried in a lossy half space, for a scalar and two dimensional case. First, the formulation of the exact model of the electromagnetic scattering is given. Then, the linear inverse problem is solved by resorting to the well assessed Singular Value Decomposition tool. The reliability of the solution procedure is tested with synthetic data achieved by a FDTD code.
\end{abstract}

Key words ground penetrating radar - magnetic anomalies - electromagnetic scattering - microwave tomography - linear inverse scattering

\section{Introduction}

Historically, electromagnetic inverse scattering has been focused much more on dielectric anomalies than on magnetic anomalies (Colton and Kress, 1992; Chew, 1995). This is because in the microwave range «to deal with» a dielectric contrast is certainly more common than «to deal with» a magnetic contrast.

However, in some cases magnetic anomalies can occur too. This can happen, for example, in the case of buried ceramic pipes or buried brick walls. Also, the study presented in this paper is of interest in the diagnostics of new materials being invented nowadays, some of which show magnetic properties in the microwave range (Jarvis et al., 2004; Chen et al., 2005).

Mailing address: Dr. Raffaele Persico, Istituto per i Beni Archeologici e Monumentali (IBAM-CNR), Via per Monteroni, Campus Universitario, 73100 Lecce, Italy; email: r.persico@ibam.cnr.it
Moreover, in some situations the soil itself exhibits magnetic more than electric properties. This occurs in presence of some magnetic minerals (Nabighian, 1987) such as magnetite, hematite, maghemite and/or iron in its free state, or when the soil is strongly polluted by industrial contaminants.

Finally, the very recent development of the Mars exploration programmes, such as MARSIS and SHARAD, has aroused a significant interest in the influence of magnetic minerals on the loss and propagation characteristics of electromagnetic waves in the GPR frequency range (Stillman and Olhoeft, 2004, 2006).

For this reason, the scattering from electric and magnetic anomalies in an electric and magnetic soil is also worth studying. Some recent works have dealt with inverse scattering from magnetic anomalies. In particular, Gustafsson and $\mathrm{He}$ (2000) tackled the two dimensional inverse scattering problem related to anomalies that show both dielectric and magnetic contrast starting from both the measurement of the electric and magnetic scattered field. In addition, Abubakar and van der Berg (2004) addressed the homologous three dimensional problem. Computational problems related to the forward model were dealt with in Nie et al. (2006). However, Gustafsson and He (2000) and Nie et al. (2006) 
refer to the case of objects embedded in a homogeneous medium, and Gustafsson and $\mathrm{He}$ (2000) and, Abubakar and van den Berg (2004) cast the inverse problem by assuming as datum both the electrical and magnetic scattered fields.

Unlike from the papers mentioned above, here we consider a half space two-dimensional geometry and assume as datum of the problem only the scattered electric field. This choice is justified because in GPR prospecting one essentially gathers a quantity roughly proportional to the electric field in the observation point.

The paper is organised as follows. Section II introduces the scattering equations relative to the «magnetic» two dimensional scalar scattering operator (providing all the relevant calculations in Appendix). The same section also proposes an inversion algorithm based on a linear model of electromagnetic scattering. Such a simplified model is similar to the well known Born model widely exploited for dielectric objects (Crocco and Soldovieri, 2003; Soldovieri et al., 2007). Section III presents numerical examples where the linear inversion is exploited to process exact scattered field data provided by means of an FDTD code. Finally, conclusions follow.

\section{Formulation of the problem}

The 2D reference scenario is composed of two homogeneous half spaces, separated by a planar interface at $z=0$ (fig. 1). The upper half space is free space, whereas the lower half space is characterized by a relative dielectric permittivity $\varepsilon_{s}$ and by a relative magnetic permeability $\mu_{\mathrm{s}}$. Both $\varepsilon_{s}$ and $\mu_{\mathrm{s}}$ can be complex to account for losses.

We consider the forward and inverse scattering problems in frequency domain. The source is assumed to be infinitely long and invariant along the $y$-axis. The considered measurement configuration is multi-bistatic (i.e. we consider a $B$ scan prospecting with a fixed offset between the transmitting and the receiving antennas) within a prefixed band of frequencies $\Omega=\left[\omega_{\min }, \omega_{\max }\right]$. The source-observation point ranges within the observation domain $\Sigma=\left[-x_{M}, x_{M}\right]$ located at the air/soil interface $(z=0)$.

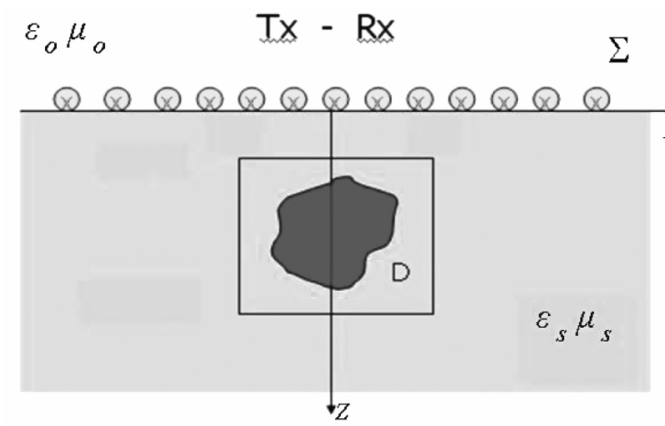

Fig. 1. Geometry of the problem for the two dimensional case and for the adopted multi-bistatic configuration

The objects are assumed infinitely long along the $y$-axis, embedded in the lower half space and they are assumed to be located in the investigation domain $D=[-a, a] \times\left[z_{\min }, z_{\min }+2 b\right]$.

Our aim is to obtain a spatial map of the magnetic properties of the investigation domain $D$, and on the basis of this map to infer the presence, location and geometry of the magnetic anomalies. Thus, the quantity of interest is the relative magnetic permeability $\mu_{r}(x, z)$ in the domain $D$; this drives to assume, as actual unknown of the problem, the magnetic contrast function

$$
\chi_{m}(x, z)=\frac{\mu_{r}(x, z)-\mu_{s}}{\mu_{s}} .
$$

It is assumed that the magnetic contrast is different from zero only inside the investigation domain $D$. In general, the magnetic contrast depends on the frequency. Here, such a dependence will be neglected in the inversion model.

It is supposed that the buried objects do not show a meaningful electric contrast with the surrounding soil. The equations of the exact electromagnetic scattering from buried magnetic anomalies are fully derived in the Appendix. The final result is given as

$$
\begin{aligned}
& E(x, z)=E_{i n c}(x, z)+k_{s}^{2} \iint_{D} \underline{\underline{G}}_{i m}\left(x, z, x^{\prime}, z^{\prime}\right) \\
& \underline{\nabla E}\left(x^{\prime}, z^{\prime}\right) \frac{\chi_{m}\left(x^{\prime}, z^{\prime}\right)}{1+\chi_{m}\left(x^{\prime}, z^{\prime}\right)} d x^{\prime} d z^{\prime} \quad(x, z) \in D
\end{aligned}
$$




$$
\begin{aligned}
& E_{s}(x, z)=k_{s}^{2} \iint_{D} \underline{\underline{G}}_{e m}\left(x, z, x^{\prime}, z^{\prime}\right) \underline{\nabla E}\left(x^{\prime}, z^{\prime}\right) \\
& \frac{\chi_{m}\left(x^{\prime}, z^{\prime}\right)}{1+\chi_{m}\left(x^{\prime}, z^{\prime}\right)} d x^{\prime} d z^{\prime} \quad(x, z) \in \sum
\end{aligned}
$$

where $k_{s}$ is the wave-number in the soil (lower half-space).

Equation (2.2) accounts for the total electric field inside the investigation domain $D$ and is given as the sum of two contributions: the incident field (i.e., the field in the investigation domain $D$ in absence of the magnetic anomalies) and the field due to the presence of scattering objects in the investigation domain. Thus $\underline{G}_{i m}=\left[G_{i m x}, G_{i m z}\right]$ denotes the internal magnetic dyadic Green's function (row vector of two elements) where

$$
\begin{aligned}
& G_{i m x}=-\frac{1}{4 \pi} \int_{-\infty}^{+\infty} \frac{u}{k_{z s} k_{s}^{2}}\left(\exp \left(-j k_{z s}(u)\left|z-z^{\prime}\right|\right)+\right. \\
& \left.+\frac{\mu_{o} k_{z s}(u)-\mu_{s} k_{z o}(u)}{\mu_{o} k_{z s}(u)+\mu_{s} k_{z o}(u)} \exp \left(-j k_{z s}(u)\left(z+z^{\prime}\right)\right)\right) \\
& \exp \left(j u\left(x-x^{\prime}\right)\right) d u
\end{aligned}
$$

$$
\begin{aligned}
& G_{i m z}=\frac{1}{4 \pi} \int_{-\infty}^{+\infty} \frac{1}{k_{s}^{2}}\left(\operatorname{sign}\left(z-z^{\prime}\right) \exp (-\right. \\
& \left.-j k_{z s}(u)\left|z-z^{\prime}\right|\right)+\frac{\mu_{s} k_{z o}(u)-\mu_{o} k_{z s}(u)}{\mu_{s} k_{z o}(u)+\mu_{o} k_{z s}(u)} \\
& \left.\exp \left(-j k_{z s}(u)\left(z+z^{\prime}\right)\right)\right) \\
& \exp \left(j u\left(x-x^{\prime}\right)\right) d u
\end{aligned}
$$

Equation (2.3) accounts for the scattered electric field on the measurement domain $\Sigma$, that represents the datum of an inverse scattering problem. The scattered field can be regarded as the electric field radiated by a magnetic current density. This magnetic current is related to the product of the gradient of the electric field inside the investigation domain times the function $\chi_{m}\left(x^{\prime}, z^{\prime}\right) /\left(1+\chi_{m}\left(x^{\prime}, z^{\prime}\right)\right)$ involving the unknown contrast. The $\underline{G}_{\text {eim }}=\left[G_{e m x}, G_{e i \mathrm{mz}}\right]$ denotes the external magnetic Green's function (row vector of two elements) where

$$
\begin{aligned}
& G_{e m x}=-\frac{1}{2 \pi} \int_{-\infty}^{+\infty} \frac{\varepsilon_{o} \mu_{o} \exp \left(j k_{z o}(u) z\right) \exp \left(-j k_{z s}(u) z^{\prime}\right)}{k_{s}^{2} \varepsilon_{s}\left(\mu_{o} k_{z s}(u)+\mu_{s} k_{z o}(u)\right)} \\
& u \exp \left(j u\left(x-x^{\prime}\right)\right) d u \\
& G_{e m z}=-\frac{1}{2 \pi} \int_{-\infty}^{+\infty} \frac{\varepsilon_{o} \mu_{o} \exp \left(j k_{z o}(u) z\right) \exp \left(-j k_{z s}(u) z^{\prime}\right)}{k_{s}^{2} \varepsilon_{s}\left(\mu_{o} k_{z s}(u)+\mu_{s} k_{z o}(u)\right)} \\
& k_{z s}(u) \exp \left(j u\left(x-x^{\prime}\right)\right) d u
\end{aligned}
$$

The gradient vector of the total field is defined in terms of the unique component of the electric total field. In formulas, this gradient is defined as

$$
\nabla E=\left(\begin{array}{l}
\frac{\partial E}{\partial x^{\prime}} \\
\frac{\partial E}{\partial z^{\prime}}
\end{array}\right)
$$

Similarly to the more widely studied case of dielectric buried anomalies (Crocco and Soldovieri, 2003; Soldovieri et al., 2007), the scattering eqs. (2.2) and (2.3) make the inverse scattering problem non-linear and ill-posed.

In order to cope with this difficulty, we adopt a simplified model of the scattering that allows us to deal with a linear inverse scattering problem. The linearization will prevent false solutions, intrinsically related to nonlinearity, and will ensure the stability of the solution by adopting well assessed regularization schemes based on the Singular Value Decomposition (Bertero and Boccacci, 1998). Conversely, the adoption of the linear model of the electromagnetic scattering, analogously to the case of reconstruction of dielectric anomalies, does not achieve a quantitative reconstruction of the buried objects but only retrieves information about their location, size and (approximately) shape.

In order to achieve a linear model for the problem at hand, we assume the hypothesis of low contrast levels $\left|\chi_{m}\right|<<1$. In this case we have

$$
\frac{\chi_{m}\left(x^{\prime}, z^{\prime}\right)}{1+\chi_{m}\left(x^{\prime}, z^{\prime}\right)} \cong \chi_{m}\left(x^{\prime}, z^{\prime}\right)
$$

and

$$
E(x, z) \cong E_{\text {inc }}(x, z)
$$

i.e. the total field inside the investigation domain can be approximated with the incident 
field. The approximation (2.8) can be «physically» justified by stating that the objects are small perturbations with respect to the host medium.

At this point, we make the further assumption that

$$
\underline{\nabla E}\left(x^{\prime}, z^{\prime}\right) \cong \underline{\nabla E_{\text {inc }}}\left(x^{\prime}, z^{\prime}\right)
$$

Let us state that eq. (2.9) cannot be straightforwardly inferred from eq. (2.8). However, in the framework of low level contrast, eq. (2.9) essentially amounts to assuming some smoothness properties of the incident field. Such an assumption is an increasingly reasonable hypothesis as far as the point in the investigation domain is farther and farther from the transmitting antenna.

Now, by substituting eq. (2.7) and eq. (2.9) in eq. (2.3), we obtain the linear inverse scattering model given by

$$
\begin{aligned}
& E_{s}(x, z)=k_{s}^{2} \iint_{D} \underline{\underline{G}} \underline{\underline{G}} \underline{\nabla E_{i n c}}\left(x^{\prime}, z^{\prime}\right) \\
& \chi_{m}\left(x^{\prime}, z^{\prime}\right) d x^{\prime} d z^{\prime} \quad z \in \Sigma
\end{aligned}
$$

Thus, the problem at hand is reduced to the inversion of the linear integral relation (2.10), where $E_{s}$ represents the datum of the problem while $\chi_{m}$ is its unknown.

In order to completely specify the integral relationship (2.10), we still have to specify the incident field. Since the primary source is a filamentary current, the incident field can be calculated in a fashion analogous to the calculation of the Green's functions provided in the Appendix.

The result is

$$
\begin{aligned}
& E_{\text {inc }}\left(x_{s}, z, x^{\prime}, z^{\prime}\right)=-f \mu_{o} \mu_{S} I_{o} \\
& \int_{-\infty}^{+\infty} \frac{\exp \left(-j k_{z s}(v) z^{\prime}\right) \exp \left(j k_{z o}(v) z\right)}{\left(\mu_{s} k_{z o}(v)+\mu_{o} k_{z s}(v)\right)} \\
& \exp \left(j v\left(x_{S}-x^{\prime}\right)\right) d v
\end{aligned}
$$

where $f$ is the frequency and $I_{o}$ is the level of the current. Thus, the gradient vector of the incident field is given by

$$
\begin{aligned}
& E_{\text {inc }}=-f \mu_{o} \mu_{S} I_{o} \\
& \int_{-\infty}^{+\infty} \frac{\exp \left(-j k_{z s}(v) z^{\prime}\right) \exp \left(j k_{z o}(v) z\right)}{\left(\mu_{o} k_{z s}(v)+\mu_{s} k_{z o}(v)\right)} . \\
& \left(\begin{array}{c}
-j v \\
-j k_{\text {ZS }}(v)
\end{array}\right) \exp \left(j v\left(x_{S}-x^{\prime}\right)\right) d v
\end{aligned}
$$

\section{Numerical results}

This section shows some numerical results to back-up the previous formulation. The synthetic scattered field data have been obtained by means of the FDTD code GPRMAX (Giannopulos, 2003). Therefore, the code for the data is totally independent from the inversion code. In particular, GPRMAX provides total field data in time domain, therefore we have to preprocess these data to obtain scattered field data in the frequency domain. The pre-processing essentially consists of muting the first part of the traces, relative to the answer to the air-soil interface and to Fourier transform the traces after this muting (Soldovieri et al., 2006). The zero time is chosen in the first maximum of the simulated traces.

Equation (2.10) has been discretized by exploiting Methods of Moments (Collin, 1985). In particular a point matching is considered in data space, while the magnetic contrast function has been represented thanks to a functional basis made up of Fourier harmonics along the horizontal direction ( $x$-direction) and pulse function along the depth ( $z$-direction).

The inversion of the linear system obtained from the discretization of eq. (2.10) has been performed thanks to the Truncated SVD (TSVD) (Bertero and Boccacci, 1998) that achieves a stable solution of the problem.

In a first example, we considered a square object with sides of $0.2 \mathrm{~m}$, buried in a soil at the depth of $1.7 \mathrm{~m}$ (referred to the upper side). The data are gathered in air at $0.01 \mathrm{~m}$ from the interface with a spatial step of $0.02 \mathrm{~m}$ and an offset between the transmitting and receiving antennas of $0.1 \mathrm{~m}$. The observation line from the first to the last source point is $1.88 \mathrm{~m}$ long, so that we have 95 GPR traces. The investigation domain is $1.98 \mathrm{~m}$ large and $2 \mathrm{~m}$ deep, and starts 


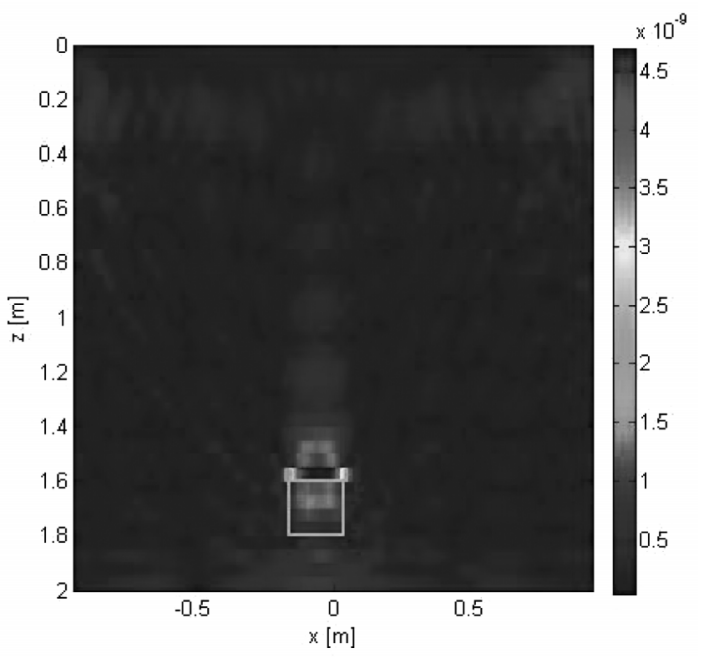

Fig. 2. Modulus of the retrieved contrast function for a square buried magnetic object at the depth of $1.7 \mathrm{~m}$. The reconstruction essentially images the upper side of the object.

from the air-soil interface. The work frequency band ranges from $200 \mathrm{MHz}$ to $1 \mathrm{GHz}$ with a frequency step of $25 \mathrm{MHz}$. The soil exhibits a relative dielectric permittivity equal to 9 and a conductivity equal to $0.01 \mathrm{~S} / \mathrm{m}$. which corresponds to a complex equivalent permittivity equal to $\varepsilon_{r}=9-j\left(0.01 / 2 \pi f \varepsilon_{o}\right)$, with $\varepsilon_{o}=8.854 \times$ $\times 10^{-12} \mathrm{~F} / \mathrm{m}$ being the dielectric permittivity of the free space. The relative magnetic permeability of the soil is $\mu_{s}=1$. The homogeneous buried object shows the same equivalent permittivity of the soil, whereas its relative magnetic permeability is $\mu_{r}=5$.

We added a white gaussian noise to the synthetic data, so that the signal to noise ratio for the total field was $20 \mathrm{~dB}$.

For the inversion scheme, we chose to retain in the TSVD expansion only the terms for which the singular values were larger than 0.01 times the maximum singular value.

Figure 2 depicts the amplitude of the retrieved contrast function normalized with respect to its maximum; the reconstruction is compared with the actual object depicted with a solid line. The tomographic reconstruction ac- curately locates the upper side of the object and determines its horizontal extent.

In order to show possible effects of the shape and mutual interactions between the objects, we propose a further example where the parameters are unchanged with respect to the previous one except that we have two circular buried objects instead of one square target. The two circular objects are buried at $0.7 \mathrm{~m}$ (with respect to their centres), their radius is $0.1 \mathrm{~cm}$ and the distance between the centres is $0.25 \mathrm{~m}$.

The reconstruction is now obtained by considering in TSVD expansion the singular values larger than 0.1 the maximum singular value.

The tomographic reconstruction is shown in fig. 3 and compared to the actual objects. Since the objects are shallower than the previous case, this time we reconstruct both the upper and lower parts of them. However, the reconstruction of the lower parts is deeper than the actual bottom of the objects because the probing wave propagates in the object more slowly than in the surrounding soil, and this is not accounted for

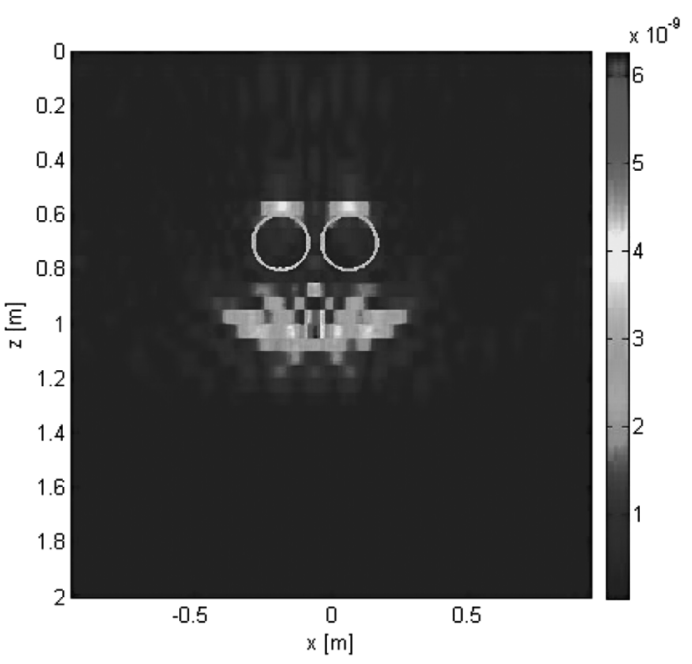

Fig. 3. Modulus of the retrieved contrast function for two circular buried magnetic objects at the depth of $0.7 \mathrm{~m}$. The reconstruction essentially images the upper side of the objects and the spot at the dept of 1 $\mathrm{m}$ arises for the mutual interactions between the two objects. 
within a linear model (Crocco and Soldovieri, 2003; Soldovieri et al., 2007). Moreover, the mutual electromagnetic interferences (not accounted by a linear model) between the two objects add constructively in a point at the depth of about one meter thereby creating an artefact.

Finally, let us stress that the adoption of the two different thresholds in TSVD (0.01 for the first case and 0.1 for the second case) makes it possible to investigate regions at different depth in the investigation domain. In particular, by lowering the TSVD threshold, we have the possibility to exploit in the reconstruction a larger number of singular functions. As a matter of fact, this allows us to image deeper objects because the «support» of higher order singular functions are located at progressively increasing depth.

\section{Conclusions}

This paper has dealt with the scattering from magnetic buried anomalies. This topic has been rarely addressed in literature, where in most cases it is assumed that both the soil and the buried objects do not have any magnetic property. Some times, however, one can meet a situation where the soil or the buried objects or both can have meaningful magnetic properties. As future developments we are working on a complete model of the 2D scattering from buried objects that show both dielectric and magnetic properties. Moreover, we will propose an analysis of the errors related to the fact that one may meet a magnetic object while looking for dielectric objects or, conversely, a dielectric object while looking for magnetic ones.

As further future developments, we would also like to address the question of the choice of the type of basis functions exploited to represent the unknowns. In fact, this choice affects both the numerical efficiency of the solution algorithm and the accuracy in the representation of the singular functions characterizing the linear operator to be inverted.

Finally, let us stress that this paper is only the first step towards the application of the approach also in realistic situations: experimental validations are in order.

\section{Appendix}

This appendix works out the mathematical formulation of the 2D scattering equations for magnetic anomalies, the source being constituted by a filamentary electrical current (the reference geometry is in fig. 1).

Let us start from Maxwell's equations

$$
\left\{\begin{array}{l}
\underline{\nabla} \times \underline{E}=-j \omega \mu \underline{H} \\
\underline{\nabla} \times \underline{H}=j \omega \varepsilon \underline{E}+\underline{J_{0}} \\
\underline{\nabla} \cdot \varepsilon \underline{E}=\rho_{0} \\
\underline{\nabla} \cdot \mu \underline{H}=0
\end{array}\right.
$$

and let us write the total fields as the superposition of the incident (unperturbed) fields and of the scattered fields.

$$
\begin{aligned}
& \underline{E}=\underline{E}_{i n c}+\underline{E}_{S} \\
& \underline{H}=\underline{H}_{i n c}+\underline{H}_{S} .
\end{aligned}
$$

Due to the two-dimensional geometry and to the kind of source, we know a priori that the electrical 
field is directed along the invariance direction, whereas the magnetic field is orthogonal to it. With respect to the reference system of fig. 1, therefore, we have

$$
\begin{aligned}
& \underline{E_{s}}=E_{s y}(x, z) i_{y} \\
& \underline{H_{s}}=H_{s x}(x, z) i_{x}+H_{s z}(x, z) i_{z} .
\end{aligned}
$$

By substitution of eq. (A.2) and eq. (A.3) in eq. (A.1), after some straightforward passages, we obtain Maxwell equations for the scattered fields

$$
\left\{\begin{array}{l}
\underline{\nabla} \times \underline{E}_{s}=-j \omega \mu_{b} \underline{H}_{s}-\underline{J}_{m e q} \\
\underline{\nabla} \times \underline{H}_{s}=j \omega \varepsilon_{b} \underline{E}_{s} \\
\underline{\nabla} \cdot \underline{E}_{s}=0 \\
\underline{\nabla} \cdot \underline{H}_{s}=-\frac{1}{j \omega \mu_{b}} \nabla \cdot J_{m e q}
\end{array}\right.
$$

being

$$
J_{\text {meq }}=j \omega \Delta \mu \underline{H}
$$

where $\Delta \mu$ is the difference between the object and the background magnetic permeability.

From the third equation in (A.5), we can rewrite the scattered electric field by means of a potential vector (also reported as Fitzgerald vector; Collin, 1985) $\underline{F}$ as

$$
\underline{E}_{s}=\frac{1}{\varepsilon_{b}} \underline{\nabla} \times \underline{F}
$$

This equation also means that, in general, we can write the potential vector as

$$
\underline{F}=F_{x}(x, z) i_{x}+F_{z}(x, z) i_{z}
$$

Substituting (A.7) in the second of Maxwell eq. (A.5) we have

$$
\underline{\nabla} \times(\underline{H}-j \omega \underline{F})=0
$$

Therefore, we can express the vector $\left(\underline{H}_{s}-j \omega \underline{F}\right)$ as the gradient of a scalar potential function $\Phi$, and so we have

$$
\underline{H}_{s}-j \omega \underline{F}=\nabla \Phi \Rightarrow \underline{H}_{s}=j \omega \underline{F}+\nabla \Phi
$$

By substitution of this equation in the first of Maxwell eq. (A.5), we obtain.

$$
\underline{\nabla} \times \frac{1}{\varepsilon_{b}} \underline{\nabla} \times \underline{F}=-j \omega \mu_{b}(j \omega \underline{F}+\nabla \Phi)-\underline{J}_{m e q}
$$

from which, by means of a well known vector identity (Collin, 1985) we have

$$
\underline{\nabla}(\nabla \cdot \underline{F})-\nabla^{2} F=k_{b}^{2} \underline{F}-j \omega \varepsilon_{b} \mu_{b} \nabla \Phi-\varepsilon_{b} \underline{J}_{m e q}
$$

$k_{b}$ being the wavenumber of the background medium, equal to

$$
k_{b}=\omega \sqrt{\mu_{b} \varepsilon_{b}}=\left\{\begin{array}{l}
k_{0}=\omega \sqrt{\mu_{0} \varepsilon_{0}} z \in \sum \\
k_{s}=\omega \sqrt{\mu_{s} \varepsilon_{s}} z \in D\left(\operatorname{Im}\left(k_{s}\right) \leq 0\right)
\end{array}\right.
$$


where $k_{o}$ and $k_{s}$ are the wave number in the free space and in the lower half space, respectively. From eq. (A.12) we have

$$
\nabla^{2} \underline{F}+k_{b}^{2} \underline{F}=\nabla\left(\underline{\nabla} \underline{F}+j \omega \mu_{b} \varepsilon_{b} \Phi\right)+\varepsilon_{b} \underline{J}_{m e q}
$$

At this point, by exploiting the gauge of Lorentz (Collin, 1985), we can impose

$$
\underline{\nabla} \underline{F}+j \omega \mu_{b} \varepsilon_{b} \Phi=0
$$

so that eq. (A.14) evolves in

$$
\nabla^{2} \underline{F}+k_{b}^{2} \underline{F}=\varepsilon_{b} \underline{J}_{\text {meq }} .
$$

Moreover, eq. (A.15) substituted in eq. (A.10) arises the expression of the magnetic scattered field vs. the only potential vector

$$
\underline{H}_{s}=j \omega \underline{F}-\frac{\nabla(\underline{\nabla} \cdot \underline{F})}{j \omega \varepsilon_{b}} .
$$

At this point, the problem is recast as the resolution of Helmotz eq. (A.16) with radiating boundary conditions at infinity. In order to solve it, let us begin by writing eq. (A.16) along its components.

$$
\begin{aligned}
& \frac{\partial^{2} F_{x}}{\partial x^{2}}+\frac{\partial^{2} F_{x}}{\partial z^{2}}+k_{b}^{2} F_{x}=\varepsilon_{b} J_{\text {meq } x} \\
& \frac{\partial^{2} F_{z}}{\partial x^{2}}+\frac{\partial^{2} F_{z}}{\partial z^{2}}+k_{b}^{2} F_{z}=\varepsilon_{b} J_{\text {meqz }} .
\end{aligned}
$$

In order to solve the problem, as is customary, we consider the Fourier transform of all quantities along the $x$-axis. The adopted convention is

$$
\hat{F}_{i}(u, z)=\int_{-\infty}^{+\infty} F_{i}(x, z) \exp (-j u x) d x \quad i=x, z
$$

Therefore, in the transformed domain $(u, z)$ eqs. (A.18) are rewritten as

$$
\begin{aligned}
& \frac{\partial^{2} \hat{F}_{x}}{\partial z^{2}}+k_{z b}^{2}(u) \hat{F}_{x}=\varepsilon_{b} \hat{J}_{m e q x} \\
& \frac{\partial^{2} \hat{F}_{z}}{\partial z^{2}}+k_{z b}^{2}(u) \hat{F}_{z}=\varepsilon_{b} \hat{J}_{\text {meqz }} .
\end{aligned}
$$

being

$$
k_{z b}=\sqrt{k_{b}^{2}-u^{2}}=\left\{\begin{array}{ll}
k_{z 0}=\sqrt{k_{0}^{2}-u^{2}} & z \in \sum \\
k_{z s}=\sqrt{k_{s}^{2}-u^{2}} & z \in D
\end{array} .\right.
$$

The imaginary part of the square roots in eqs. (A.21) is meant to be negative to ensure that the amplitude of the fields vanishes far from the sources.

Since eqs. (A.20) are linear, we can solve them by adding the solutions of suitable impulsive answers, as it is well known, therefore, let us begin to consider impulsive sources placed in some generic (buried) point $\left(x^{\prime}, z^{\prime}\right)$ :

$$
\begin{aligned}
& J_{\text {meqx }}(x, z)=I_{\text {meqx }} \delta\left(x-x^{\prime}\right) \delta\left(z-z^{\prime}\right) \\
& J_{\text {meqz }}(x, z)=I_{\text {meqz }} \delta\left(x-x^{\prime}\right) \delta\left(z-z^{\prime}\right)
\end{aligned}
$$


Which in the transformed domain becomes

$$
\begin{aligned}
& \hat{J}_{\text {meqx }}(u, z)=I_{\text {meqx }} \exp \left(-j u x^{\prime}\right) \delta\left(z-z^{\prime}\right) \\
& \hat{J}_{\text {meqz }}(u, z)=I_{\text {meqz }} \exp \left(-j u x^{\prime}\right) \delta\left(z-z^{\prime}\right)
\end{aligned}
$$

with this sources, eqs. (A.20) are recast as

$$
\begin{aligned}
& \frac{\partial^{2} \hat{F}_{x}}{\partial z^{2}}+k_{z b}^{2}(u) \hat{F}_{x}=\varepsilon_{b} I_{m e q x} \exp \left(-j u x^{\prime}\right) \delta\left(z-z^{\prime}\right) \\
& \frac{\partial^{2} \hat{F}_{z}}{\partial z^{2}}+k_{z b}^{2}(u) \hat{F}_{z}=\varepsilon_{b} I_{\text {meqz }} \exp \left(-j u x^{\prime}\right) \delta\left(z-z^{\prime}\right)
\end{aligned}
$$

Because of the radiation condition at infinity (Collin, 1985), both (A.24) equations have a general solution that can be written as

$$
\hat{F}_{i}=\left\{\begin{array}{l}
A_{i}(u) \exp \left(j k_{z 0}(u) z\right) z<0 \\
B_{i}(u) \exp \left(j k_{z s}(u) z\right)+C_{i}(u) \exp \left(-j k_{z s}(u) z\right) 0<z<z^{\prime}(i=x, z) \\
D_{i}(u) \exp \left(-j k_{z s}(u) z\right) z^{\prime}<z
\end{array}\right.
$$

In order to find the four functions $A, B, C$ and $D$ we have to impose four conditions. These are given as:

1) Continuity of the tangential component of the electric field at the air-soil interface.

2) Continuity of the tangential component of the magnetic field at the air-soil interface.

3) Continuity of the potential vector at the depth $z^{\prime}$ of the source

4) Integrability of the source and of the potential vector (i.e. of eqs. (A.24)) about the depth of the source.

An important point is the fact that we can look for a solution parallel to the source both with respect to the $x$-component and to the $z$-component of the magnetic current density, i.e. we can solve for two uncoupled problems synthesized as follows:

$$
\begin{aligned}
& F=F_{x} i_{x} \Longleftrightarrow \hat{J}_{\text {meq }}=\hat{J}_{\text {meqx }} i_{x} \\
& F=F_{z} i_{z} \Longleftrightarrow \hat{J}_{\text {meq }}=\hat{J}_{\text {meqz }} i_{z}
\end{aligned}
$$

Thanks to this (not trivial) fact, we can retrieve the eight quantities $A_{x}, B_{x}, C_{x}, D_{x}, A_{z}, B_{z}, C_{z}, D_{z}$, from two uncoupled linear systems The calculations are long but straightforward, and the procedure is quite known. Therefore, we can limit to provide the final results of these passages, that are

$$
\hat{F}_{x}=\left\{\begin{array}{l}
\frac{j \varepsilon_{o} \mu_{o} k_{z s}(u) I_{\text {meqx }} \exp \left(-j u x^{\prime}\right)}{k_{z o}\left(\mu_{o} k_{z s}(u)+\mu_{s} k_{z o}(u)\right)} \exp \left(j k_{z o}(u) z\right) \exp \left(-j k_{z s}(u) z^{\prime}\right) z<0 \\
\frac{j \varepsilon_{s} I_{m e q x} \exp \left(-j u x^{\prime}\right)}{2 k_{z s}(u)}\left[\exp \left(j k_{z s}(u)\left(z-z^{\prime}\right)\right)+\left(\frac{\mu_{s} k_{z o}(u)-\mu_{o} k_{z s}(u)}{\mu_{s} k_{z o}(u)+\mu_{o} k_{z s}(u)}\right) \exp \left(-j k_{z s}(u)\left(z+z^{\prime}\right)\right)\right] 0<z<z^{\prime} \\
\frac{j \varepsilon_{s} I_{\text {meqx }} \exp \left(-j u x^{\prime}\right)}{2 k_{z s}(u)}\left[\exp \left(-j k_{z s}(u)\left(z-z^{\prime}\right)\right)+\left(\frac{\mu_{s} k_{z o}(u)-\mu_{o} k_{z s}(u)}{\mu_{s} k_{z o}(u)+\mu_{o} k_{z s}(u)}\right) \exp \left(-j k_{z s}(u)\left(z+z^{\prime}\right)\right)\right] z^{\prime}<z
\end{array}\right.
$$


and

$$
\hat{F}_{z}=\left\{\begin{array}{l}
\frac{j \varepsilon_{o} \mu_{o} I_{m e q z} \exp \left(-j u x^{\prime}\right)}{\mu_{o} k_{z s}(u)+\mu_{s} k_{z o}(u)} \exp \left(-j k_{z s}(u) z\right) \exp \left(j k_{z o}(u) z^{\prime}\right) z<0 \\
\frac{j \varepsilon_{s} I_{m e q z} \exp \left(-j u x^{\prime}\right)}{2 k_{z s}(u)}\left[\exp \left(j k_{z s}(u)\left(z-z^{\prime}\right)\right)+\left(\frac{\mu_{o} k_{z s}(u)-\mu_{s} k_{z o}(u)}{\mu_{o} k_{z s}(u)+\mu_{s} k_{z o}(u)}\right) \exp \left(-j k_{z s}(u)\left(z+z^{\prime}\right)\right)\right] 0<z<z^{\prime} \\
\frac{j \varepsilon_{s} I_{m e q z} \exp \left(-j u x^{\prime}\right)}{2 k_{z s}(u)}\left[\exp \left(-j k_{z s}(u)\left(z-z^{\prime}\right)\right)+\left(\frac{\mu_{o} k_{z s}(u)-\mu_{s} k_{z o}(u)}{\mu_{o} k_{z s}(u)+\mu_{s} k_{z o}(u)}\right) \exp \left(-j k_{z s}(u)\left(z+z^{\prime}\right)\right)\right] z^{\prime}<z
\end{array}\right.
$$

From the potential vector, we can express the scattered electric field thanks to eq. (A.7), being the curl meant in the transformed domain. The result is (after some further straightforward passages)

$$
\hat{E}_{s y}=\left\{\begin{array}{l}
-\frac{\varepsilon_{o} \mu_{o} k_{z s}(u) I_{\text {meqx }} \exp \left(-j u x^{\prime}\right)}{\varepsilon_{s}\left(\mu_{o} k_{z s}(u)+\mu_{s} k_{z o}(u)\right)} \exp \left(-j k_{z s}(u) z^{\prime}\right) \exp \left(j k_{z o}(u) z\right)+ \\
+\frac{u \varepsilon_{o} \mu_{o} I_{\text {meqz }} \exp \left(-j u x^{\prime}\right)}{\varepsilon_{s}\left(\mu_{o} k_{z s}(u)+\mu_{s} k_{z o}(u)\right)} \exp \left(-j k_{z s}(u) z^{\prime}\right) \exp \left(j k_{z o} z\right) \quad z \in \sum \\
\frac{I_{m e q z} \exp \left(-j u x^{\prime}\right)}{2}\left[\operatorname{sign}\left(z-z^{\prime}\right) \exp \left(-j k_{z s}(u)\left|z-z^{\prime}\right|\right)+\left(\frac{\mu_{s} k_{z o}(u)-\mu_{o} k_{z s}(u)}{\mu_{s} k_{z o}(u)+\mu_{o} k_{z s}(u)}\right) \exp \left(-j k_{z s}(u)\left(z+z^{\prime}\right)\right)\right]+ \\
+\frac{u I_{\text {meqz }} \exp \left(-j u x^{\prime}\right)}{2 k_{z s}(u)}\left[\exp \left(-j k_{z s}(u) \mid z-z^{\prime}\right)+\left(\frac{\mu_{o} k_{z s}(u)-\mu_{s} k_{z o}(u)}{\mu_{o} k_{z s}(u)+\mu_{s} k_{z o}(u)}\right) \exp \left(-j k_{z s}(u)\left(z+z^{\prime}\right)\right)\right] z \in D
\end{array}\right.
$$

This is the solution in the transformed domain and for an impulsive source. In order to pass to the distributed solution relevant to the case at hand, we express eq. (A.6) versus the scattered field and along its components

$$
\begin{aligned}
& J_{\text {meqx }}\left(x^{\prime}, z^{\prime}\right)=j \omega \Delta \mu H_{x}\left(x^{\prime}, z^{\prime}\right)=j \omega \Delta \mu \frac{1}{j \omega \mu} \frac{\partial E_{y}\left(x^{\prime}, z^{\prime}\right)}{\partial z^{\prime}}=\frac{\frac{\Delta \mu}{\mu_{b}}}{\frac{\mu_{b}+\Delta \mu}{\mu_{b}}} \frac{\partial E_{y}\left(x^{\prime}, z^{\prime}\right)}{\partial z^{\prime}}= \\
& =\frac{\chi_{m}\left(x^{\prime}, z^{\prime}\right)}{1+\chi_{m}\left(x^{\prime}, z^{\prime}\right)} \frac{\partial E_{y}\left(x^{\prime}, z^{\prime}\right)}{\partial z^{\prime}} \\
& J_{\text {meq }}\left(x^{\prime}, z^{\prime}\right)=j \omega \Delta \mu H_{z}\left(x^{\prime}, z^{\prime}\right)=-j \omega \Delta \mu \frac{1}{j \omega \mu} \frac{\partial E_{y}\left(x^{\prime}, z^{\prime}\right)}{\partial x^{\prime}}=\frac{\frac{\Delta \mu}{\mu_{b}}}{\frac{\mu_{b}+\Delta \mu}{\mu_{b}}} \frac{\partial E_{y}\left(x^{\prime}, z^{\prime}\right)}{\partial x^{\prime}}= \\
& =-\frac{\chi_{m}\left(x^{\prime}, z^{\prime}\right)}{1+\chi_{m}\left(x^{\prime}, z^{\prime}\right)} \frac{\partial E_{y}\left(x^{\prime}, z^{\prime}\right)}{\partial x^{\prime}}
\end{aligned}
$$

Therefore, the elementary magnetic currents to be substituted and then integrated in eq. (A.29) are given by

$$
\begin{aligned}
& I_{\text {meqx }}\left(x^{\prime}, z^{\prime}\right)=J_{\text {meqx }}\left(x^{\prime}, z^{\prime}\right) d x^{\prime} d z^{\prime}=\frac{\chi_{m}\left(x^{\prime}, z^{\prime}\right)}{1+\chi_{m}\left(x^{\prime}, z^{\prime}\right)} \frac{\partial E_{y}\left(x^{\prime}, z^{\prime}\right)}{\partial z^{\prime}} d x^{\prime} d z^{\prime} \\
& I_{\text {meqz }}\left(x^{\prime}, z^{\prime}\right)=J_{\text {meq }}\left(x^{\prime}, z^{\prime}\right) d x^{\prime} d z^{\prime}=\frac{\chi_{m}\left(x^{\prime}, z^{\prime}\right)}{1+\chi_{m}\left(x^{\prime}, z^{\prime}\right)} \frac{\partial E_{y}\left(x^{\prime}, z^{\prime}\right)}{\partial x^{\prime}} d x^{\prime} d z^{\prime}
\end{aligned}
$$

After substitution of eq. (A.31) in eq. (A.29) and after integration in the variables and in the inves- 
tigation domain $D$, the scattered field in the domain is obtained. In order to achieve the scattered field in the spatial domain an inverse Fourier transform is still required. Again, the passages are long but straightforward, therefore we only provide the final result of them

$$
\begin{aligned}
& E_{s y}(x, z)=k_{s}^{2} \iint_{D}\left(\frac{1}{2 \pi} \int_{-\infty}^{+\infty}-\frac{\varepsilon_{o} \mu_{o} k_{z s}(u) \exp \left(j k_{z o}(u) z\right) \exp \left(-j k_{z s}(u) z^{\prime}\right)}{k_{s}^{2} \varepsilon_{s}\left(\mu_{o} k_{z s}(u)+\mu_{s} k_{z o}(u)\right)} \exp \left(j u\left(x-x^{\prime}\right)\right) d u\right) \\
& \frac{\chi_{m}\left(x^{\prime}, z^{\prime}\right)}{1+\chi_{m}\left(x^{\prime}, z^{\prime}\right)} \frac{\partial E\left(x^{\prime}, z^{\prime}\right)}{\partial z^{\prime}} d x^{\prime} d z^{\prime}+ \\
& +k_{s}^{2} \iint_{D}\left(\frac{1}{2 \pi} \int_{-\infty}^{+\infty}-\frac{u \varepsilon_{o} \mu_{o}(u) \exp \left(j k_{z o}(u) z\right) \exp \left(-j k_{z s}(u) z^{\prime}\right)}{k_{s}^{2} \varepsilon_{s}\left(\mu_{o} k_{z s}(u)+\mu_{s} k_{z o}(u)\right)} \exp \left(j u\left(x-x^{\prime}\right)\right) d u\right) \\
& \frac{\chi_{m}\left(x^{\prime}, z^{\prime}\right)}{1+\chi_{m}\left(x^{\prime}, z^{\prime}\right)} \frac{\partial E\left(x^{\prime}, z^{\prime}\right)}{\partial x^{\prime}} d x^{\prime} d z^{\prime} \quad z<0
\end{aligned}
$$

in air and

$$
\begin{aligned}
& E_{s y}(x, z)=k_{s}^{2} \iint_{D}\left(\frac { 1 } { 4 \pi k _ { s } ^ { 2 } } \int _ { - \infty } ^ { + \infty } \left[\operatorname{sign}\left(z-z^{\prime}\right) \exp \left(-j k_{z s}(u)\left|z-z^{\prime}\right|\right)+\left(\frac{\mu_{s} k_{z o}(u)-\mu_{o} k_{z s}(u)}{\mu_{s} k_{z o}(u)+\mu_{o} k_{z s}(u)}\right)\right.\right. \\
& \left.\left.\exp \left(-j k_{z s}\left(z+z^{\prime}\right)\right)\right] \exp \left(j u\left(x-x^{\prime}\right)\right) d u\right) \frac{\chi_{m}\left(x^{\prime}, z^{\prime}\right)}{1+\chi_{m}\left(x^{\prime}, z^{\prime}\right)} \frac{\partial E\left(x^{\prime}, z^{\prime}\right)}{\partial z^{\prime}} d x^{\prime} d z^{\prime}+ \\
& +k_{s}^{2} \iint_{D}\left(-\frac{1}{4 \pi k_{s}^{2}} \int_{-\infty}^{+\infty} \frac{u}{k_{z s}}\left[\exp \left(-j k_{z s}(u)\left|z-z^{\prime}\right|\right)+\left(\frac{\mu_{o} k_{z s}(u)-\mu_{s} k_{z o}(u)}{\mu_{o} k_{z s}(u)+\mu_{s} k_{z o}(u)}\right)\right.\right. \\
& \left.\left.\exp \left(-j k_{z s}\left(z+z^{\prime}\right)\right)\right] \exp \left(j u\left(x-x^{\prime}\right)\right) d u\right) \frac{\chi_{m}\left(x^{\prime}, z^{\prime}\right)}{1+\chi_{m}\left(x^{\prime}, z^{\prime}\right)} \frac{\partial E\left(x^{\prime}, z^{\prime}\right)}{\partial x^{\prime}} d x^{\prime} d z^{\prime} \quad z>0
\end{aligned}
$$

in the soil.

At this point, since the total field in the investigation domain is given by the sum of the incident and the scattered field, eqs. ((A.31)-(A.33)) provide quite immediately eqs. (2.1) and (2.2) in the Section 2.

\section{REFERENCES}

ABUBAKAR, A. and P.M. vAn DEN BERG (2004): Iterative forward and inverse algorithms based on domain integral equations for three-dimensional electric and magnetic objects, J. Comput. Phys., 195, 236-262.

BERTERO, M. and P. BocCACCI (1998): Introduction to Inverse Problems in Imaging (IOP, Bristol, U.K.).

CHEw, W.C. (1995): Waves and Fields in Inhomogeneous Media (IEEE Ppress, Piscataway, N.J.).

Chen, P., R.X. Wu, T. ZhaO, F. YANG and J.Q. Xiao (2005): Complex permittivity and permeability of metallic magnetic granular composites at microwave frequencies, J. Phys. D Appl. Phys., 38, 2302-2305.

Collin, R.E. (1985): Antennas and Radiowave Propagation (McGraw-Hill).

Colton, D. and R. KRess (1992): Inverse Acoustic and Electromagnetic Scattering Theory (Springer Verlag).

Crocco, L. and F. Soldovieri (2003): A microwave tomographic approach for imaging targets buried in a layered medium, Ann. Geophys., 46 (3), 559-572.
Dural, D. and M.I. Aksun (1995): Closed-Form Green's Functions for General Sources and Stratified Media, IEEE Trans. Microwave Theory Techniques, 43 (7), 1545-1552.

Giannopulos, A. (2003): GprMax2D V 1.5 (Electromagnetic Simulator for Ground Probing Radar).

Gustafsson, M. and S. He (2000): An optimization approach to two-dimensional time domain electromagnetic inverse problems, Radio Sci., 35 (2), 525-536.

Jarvis, J.B., M.D. JANEZIC, B.F. RiddLe, R.T. JohnK, P. KABos, C.L. Holloway, R.G. Geyer and C.A. GrosvenOVER (2004): Measuring the permittivity and permeability of lossy materials: solids, liquids, metals, buildings materials, and negative-index materials, NIST Technical Note 1536.

NABIGHIAN, M. (1987): Electromagnetic Methods in Applied Geophysics-Theory (Society of Exploration Geophysics), vol. 1.

Nie, X.C., N. Yuan, L.W. Li, Y.B. Gan and T.S. YeO (2006): A fast combined field volume integral equation solution to EM scattering by $3 \mathrm{D}$ dielectric objects of 
arbitrary permittivity and permeability, IEEE Trans. Antennas Prop., 54 (3), 961-969.

Piscitelli, S., E. Rizzo, F. Cristallo, V. Lapenna, L. Crocco, R. Persico and F. Soldovieri (2007): GPR and microwave tomography for detecting shallow cavities in the historical area of Sassi of Matera (Southern italy), Near Surface Geophysics (accepted).

Soldovieri, F., R. Persico and G. LeOne (2006): A microwave tomographic imaging approach for multibistatic configuration: the choice of frequency step, IEEE Trans. Instrumentation Measurements, 55 (6), 19261934.

Soldovieri, F., J. Hugenschmidt, R. Persico and G. LeOne (2007): A linear inverse scattering algorithm for realis- tic GPR applications, Near Surface Geophys., 5 (1), $29-42$.

Stillman, D.E. and G.R. Olhoeft (2004): GPR and magnetic minerals at Mars temperatures, in Tenth International Conference on Ground Penetrating Radar, 735738.

Stillman, D.E. and G.R. OlhoEfT (2006): Electromagnetic properties of martian analog minerals at radar frequencies and martian temperatures, in Proceedings of the 37th Lunar and Planetary Science Conference.

Young, J.L. and C.M. JoHnson (2004): A Compact Recursive Trans-Impedance Green's Function for the Inhomogeneous Ferrite Microwave Circulator, IEEE Trans. Microwave Theory Techniques, 52 (7), 1751-1759. 